\title{
The Aortic Annulus, Sinus of Valsalva, Ascending Aorta and Z-score Values in Healthy Children
}

\section{(1) Şebnem Paytoncu}

Manisa City Hospital, Clinic of Pediatric Cardiology, Manisa, Turkey

\begin{abstract}
Objectives: The aim of this study is to evaluate echocardiographically (and determine pathology if there is any) the diameters and Z-scores of aortic annulus, sinus of valsalva and ascending aorta in healthy children.

Materials and Methods: This retrospective crosssectional study includes seventy patients with no important hemodynamically congenital heart disease. Forty-one of 0-18 (average 5.69) year old patients are boys (58.6\%) and 29 are girls (41.4\%). Patients' height and weight values were assessed. Body surface area and Z-score values were calculated. Aortic annulus, sinus of valsalva and ascending aorta were measured on the parasternal long axis view. The indexed values were obtained by dividing all measured parameters by body surface area.
\end{abstract}

Results: Average indexed aortic annulus value is 22.77 $\mathrm{mm} / \mathrm{m}^{2}$; sinus of valsalva value is $30.51 \mathrm{~mm} / \mathrm{m}^{2}$ and ascending aortic value is $28.30 \mathrm{~mm} / \mathrm{m}^{2}$ in our study. Average aortic annulus value for nine patients whose Z-scores are $>2$ is $26.5 \mathrm{~mm} / \mathrm{m}^{2}$; sinus of valsalva value is $38.24 \mathrm{~mm} / \mathrm{m}^{2}$ and ascending aortic value is $34.94 \mathrm{~mm} / \mathrm{m}^{2}$. Conclusion: In the present study, children aged between 0-18 years, mean aortic annulus, sinus of valsalva and ascending aorta, Z-scores and indexed values (aortic annulus $/ \mathrm{mm}^{2}$, sinus of valsalva $/ \mathrm{m}^{2}$, ascending aorta $/ \mathrm{m}^{2}$ ) were determined. In this study including few patients, aortic dilatations have been observed in the measurements of six of 67 patients $(8.9 \%)$ who visited for the first time and 9 of 70 patients in total $(12 \%)$. It is remarkable that nonsyndromic newborn and infant patients who are thought to be healthy have also various levels of aortic dilatations even before they were diagnosed with aortopathy.

Keywords: Aortopathy, aorta annulus, sinus of valsalva, ascending aorta, Z-score, children

Address for Correspondence: Şebnem Paytoncu, Manisa City Hospital, Clinic of Pediatric Cardiology, Manisa, Turkey

Phone: +90 5306906693 e-mail: sebnempaytoncu888@hotmail.com ORCID: orcid.org/0000-0003-1538-1435

Received: 22.06.2019 Accepted: 15.08.2019

Cite this article as: Paytoncu Ş. The Aortic Annulus, Sinus of Valsalva, Ascending Aorta and Z-score Values in Healthy Children. EJCM 2019;7(3):126-132.

DOI: 10.32596/ejcm.galenos.2019.06.035

Presented in: This study was presented as an oral presentation at the $15^{\text {th }}$ International Congress of Update in Cardiology and Cardiovascular surgery by the name of "The Echocardiographic Assessment of Aortic Annulus, Sinus of Valsalva and Ascending Aorta Diameters in Children" at March 28, 2019, Antalya, Turkey. 


\section{Introduction}

Aortic pathologies like hypoplasia, dilatation, aneurysm and rupture at various levels of aorta, valvular stenosis/insufficiency and coronary arterial abnormalities can be seen in children and adults. These congenital and acquired pathologies can cause serious mortality and morbidity.

Primary aortic dilatation is mostly seen with conotruncal anomalies such as bicuspid aortic valve, CoA (coarctation of aorta), Tetralogy of Fallot, PA/VSD (Pulmonary atresia/Ventricular Septal Defect), and TA (Truncus arteriosus). This is also a feature in genetic syndromes such as Marfan, Loeys-Dietz, vascular EhlersDanlos, and Turner syndromes. Secondary dilatation of the aorta is seen after congenital cardiac surgery, as in the Ross, arterial switch operation, systemic outflow tract reconstruction in single-ventricle patients. Aortic pathology can be at a single level, as well as at multiple levels, in the entire aortic wall ${ }^{(1,2)}$.

\section{Objectives}

The aim of this study is to echocardiographically evaluate (and determine pathology if there is any) the diameters and Z-scores of aortic annulus (Ao ann), sinus of valsalva (SoV) and ascending aorta (Asc ao) in healthy children.

\section{Materials and Methods}

\section{Patients}

This retrospective cross-sectional study included patients who were admitted to Pediatric Cardiology Department during the period from September 01 to October 24 in 2018. Patients with cardiac murmur, chest pain, fatigue, and palpitation were assessed with their medical histories, physical examination, ECG, and when needed, telecardiography. No sedation was applied to patients before echocardiography. This study includes 70 patients with no important hemodynamically congenital heart disease, out of 579 patients whose various levels of aortic diameters (Ao ann, SoV, Asc ao) were measured through echocardiography. Forty one of 0-18 (average $5.69)$ year-old patients were boys $(58.6 \%)$ and 29 were girls $(41.4 \%)$.Thirty one of them $(44.3 \%)$ were 0 -1-yearold, 39 of them (55.7\%) were 1-18-year-old. Patients' height and weight values were assessed and BSA (Body surface area) and Z-score values were calculated. BSA was calculated through the formula of $4 \mathrm{x}+7 / 90+\mathrm{x}$, and $\mathrm{Z}$-score values were obtained according to the report of Cantinotti et al. (3) Written informed consent for participation was obtained.

\section{Echocardiography}

Patients were assessed by the same researcher (SP) by using Philips HDXE11 Phase Array (Philips Medical System, Nederland BV, Best, the Netherlands) pediatric transducer with S8-3 and S4-2 mHz Broadband sector array transducers. Routine transthoracic echocardiographic examination was conducted according to the segmental analysis method. Aortic valve Doppler measurements were done in apical 4-chamber and suprasternal views. Ao ann, $\mathrm{SoV}$ and Asc ao measurements in consecutive cardiac beats were recorded as the average of three measurements. Ao ann was measured on the parasternal long axis view as the maximum distance between the hinge points of leaflets in the systole, and SoV and Asc ao were measured as perpendicular to the long axis of aorta and as forming maximum end diastolic diameters ${ }^{(4,5)}$. The indexed values [indexed Ao ann (iAa); indexed SoV (iSoV); indexed Asc ao (iAsc ao)] were obtained by dividing all measured parameters by BSA.

\section{Statistical Analysis}

SPSS 15.0 (SPSS for Windows v.15.0; IBM-SPSS Inc., Chicago, IL, USA) software was used for all statistical analyses. An Independent Sample t-test was used for the comparison of parametric values. Pearson correlation test and linear regression analysis were applied to evaluate the correlation among variables. The correlation coefficient which was $<0.3$ was defined as weak; $0.3-0.7$ as medium, and $>0.7$ as strong. Continuous variables were reported as mean \pm SD (standard deviation). 
As given in literature, the Z-score, a standardized value that indicates by how many SDs a value is above or below the mean in a normally distributed population, has been recommended for normalization. A measurement that is two SDs above the mean (the $97.7^{\text {th }}$ percentile) has a $\mathrm{Z}$-score of +2 , whereas a measurement that is two SDs below the mean (the $2.3^{\text {rd }}$ percentile) has a Z-score of $-2^{(6)}$.

\section{Results}

The basic characteristics and echocardiographic findings of all patients are presented in Table 1. Patients were categorized by BSA into five groups. BSA with 0.12-2.25 $\mathrm{m}^{2}$ formed group 1, and 0.251-0.5 $\mathrm{m}^{2}$ group 2, 0.51-1 $\mathrm{m}^{2}$ group 3, 1.01-1.5 $\mathrm{m}^{2}$ group 4, and 1.51-1.9 $\mathrm{m}^{2}$ group 5. Z-score and indexed aortic measurements [IAM (iAa, iSoV, iAsc ao)] are given in Tables 2-4. It was found out that there was not any significant difference of Z-score values and IAM according to gender. There was a close correlation between BSA and Ao ann $(r=0.935, p<0.001)$.

Table 1. Anthropometrşc and echocardiographic features of patients

\begin{tabular}{|c|c|c|c|c|}
\hline & Minimum & Maximum & Mean & SD \\
\hline Age (year) & 0.00 & 18.00 & 5.68 & 5.86 \\
\hline Weight (kg) & 1.49 & 79.35 & 22.23 & 20.64 \\
\hline Height (cm) & 38.00 & 179.00 & 102.36 & 46.65 \\
\hline $\operatorname{BSA}\left(\mathrm{m}^{2}\right)$ & 0.14 & 1.91 & 0.75 & 0.52 \\
\hline Z-score (weight) & -3.83 & 2.41 & -0.15 & 1.39 \\
\hline Z-score (height) & -7.45 & 4.31 & 0.11 & 1.62 \\
\hline Aortic annulus (mm) & 5.62 & 22.20 & 13.00 & 5.30 \\
\hline Sinus of valsalva $(\mathrm{mm})$ & 7.55 & 35.30 & 17.30 & 7.19 \\
\hline Ascending aorta $(\mathrm{mm})$ & 6.39 & 32.50 & 15.97 & 6.64 \\
\hline Z-score aortic annulus & -2.23 & 4.19 & 0.11 & 1.18 \\
\hline $\begin{array}{l}\text { Z-score sinus of } \\
\text { valsalva }\end{array}$ & -3.02 & 6.37 & -0.28 & 1.51 \\
\hline $\begin{array}{l}\text { Z-score ascending } \\
\text { aorta }\end{array}$ & -3.92 & 4.53 & 0.10 & 1.36 \\
\hline Aortic annulus $/ \mathrm{m}^{2}$ & 10.17 & 41.66 & 22.77 & 8.80 \\
\hline Sinus of valsalva/m² & 13.21 & 61 & 30.51 & 12.33 \\
\hline Ascending aorta/m² & 12.40 & 58.88 & 28.30 & 11.92 \\
\hline
\end{tabular}

$S D$ : Standard deviation, BSA: Body surface area
The same close correlation was also seen between BSA -SoV $(\mathrm{r}=0.886, \mathrm{p}<0.001)$ and BSA-Asc ao $(\mathrm{r}=0.921$, $\mathrm{p}<0.001)$. Similarly, close correlations were found between BSA-iAa $(r=0.82, \mathrm{p}<0.001)$, BSA-iSoV $(r=0.78$, $\mathrm{p}<0.001)$ and BSA-iAsc ao $(\mathrm{r}=0.74, \mathrm{p}<0.001)$.

Anthropometric measurements of patients were evaluated with the "Standardized Height and Weight calculator", and Z-scores of height and weight values were obtained. There was a medium and statistically meaningful correlation between z-scores of height and weight $(r=0.67, p<0.001)$.

There was a statistically significant and strong correlation between Z-scores of Ao ann and SoV ( $\mathrm{r}=0.778$, $\mathrm{p}<0.001)$. It was found out that there was a statistically meaningful and medium correlation between Z-scores of Ao ann and Asc ao ( $r=0.427, \mathrm{p}<0.001)$. A statistically significant and medium correlation between Z-scores of SoV and Asc ao $(r=0.644, p<0.001)$ were identified. A statistically significant and highly strong correlation was revealed between Ao ann and Asc Ao; the same correlation was found out between $\mathrm{SoV}$ and $\mathrm{Asc}$ ao $(\mathrm{r}=0.949, \mathrm{p}<0.001)$.

There was a statistically significant and similar strong correlation between Ao ann and SoV ( $\mathrm{r}=0.972$, $\mathrm{p}<0,001)$. Z-score values of the three patients, whose $Z$-score Ao ann values were greater than $2(>2)$, were 2.98, 2.8 and 4.19. Echocardiography of one of the patients was normal. The second patient had scoliosis, MVP (Mitral valve prolapse) and AVP (aortic valve prolapse). The third patient had scoliosis, MVP, AVP, phenotypic Marfan syndrome. No genetic pathologies were detected.

$\mathrm{Z}$-scores of the three patients, whose Z-scores of SoV values were greater than $2(>2)$, were 3.17, 2.09 and 6.37. One of the patients had hemodynamically insignificant small VSD. The other two were the patients with scoliosis, whose Z-scores of annulus values were greater than $2(>2)$.

There were six patients whose Z-score of asc ao was greater than $2(>2)$. One of them, whose $\mathrm{SoV}$ was greater 
than $2(>2)$, had small VSD with Z-scores. That patient's $Z$-score value was 4.53. The Z-scores of Asc ao values of the others were $2.18,2.3,2.48,2.67$, and 2.73. The patients whose Z-score of Asc ao values were 2.48 and 2.3 were a 1.5 -month-old girl and 3-month-old boy. The other echocardiographic findings of those patients were normal. A 17.5-year-old boy and, a 18- year-old girl were two patients with no congenital heart disease, and no pathology was associated with echocardiography except for that Z-score of Asc ao was greater than $2(>2)$. Their values were 2.73 and 2.18 , respectively. All these values were summarized in Table 5.

Table 2. Z-score values according to BSA

\begin{tabular}{|c|c|c|c|c|c|}
\hline $\operatorname{BSA}\left(m^{2}\right)$ & Group & n (\%) & $\begin{array}{l}\text { Z-score annulus } \\
\text { Mean } \pm \text { SD }\end{array}$ & $\begin{array}{l}\text { Z-score sinus of valsalva } \\
\text { Mean } \pm \text { SD }\end{array}$ & $\begin{array}{l}\text { Z-score of ascend aorta } \\
\text { Mean } \pm \text { SD }\end{array}$ \\
\hline $0-0.25$ & 1 & $22(31.46)$ & $-0.42 \pm 1.27$ & $-1.01 \pm 1.36$ & $-0.4 \pm 1.48$ \\
\hline $0.251-0.5$ & 2 & $9(12.9)$ & $0.18 \pm 0.61$ & $-0.57 \pm 0.99$ & $-0.62 \pm 0.83$ \\
\hline $0.51-1$ & 3 & $15(21.4)$ & $0.92 \pm 1.34$ & $0.78 \pm 2.02$ & $0.76 \pm 1.43$ \\
\hline $1.01-1.5$ & 4 & $9(12.9)$ & $-0.28 \pm 0.86$ & $-0.61 \pm 0.98$ & $0.49 \pm 1.44$ \\
\hline \multirow[t]{2}{*}{$1.51-1.9$} & 5 & $15(21.4)$ & $0.21 \pm 0.84$ & $0.11 \pm 1$ & $0.36 \pm 0.84$ \\
\hline & Total & 70 & $0.11 \pm 1.18$ & $-0.28 \pm 1.51$ & $0.09 \pm 1.35$ \\
\hline
\end{tabular}

SD: Standard deviation, BSA: Body surface area

Table 3. Aortic annulus, sinus of valsalva and ascending aorta values

\begin{tabular}{|c|c|c|c|c|c|}
\hline \multirow[b]{2}{*}{$\operatorname{BSA}\left(\mathrm{m}^{2}\right)$} & \multirow[b]{2}{*}{ Group } & \multirow[b]{2}{*}{ n (\%) } & \multirow{2}{*}{$\begin{array}{l}\text { Aort annulus }(\mathrm{mm}) \\
\text { Mean } \pm \text { SD }\end{array}$} & \multirow{2}{*}{$\begin{array}{l}\text { Sinus of valsalva }(\mathrm{mm}) \\
\text { Mean } \pm \text { SD }\end{array}$} & \multirow{2}{*}{$\begin{array}{l}\text { Asc Ao }(\mathrm{mm}) \\
\text { Mean } \pm \text { SD }\end{array}$} \\
\hline & & & & & \\
\hline $0-0.25$ & 1 & $22(31.46)$ & $6.89 \pm 0.91$ & $9.27 \pm 1.18$ & $8.78 \pm 1.15$ \\
\hline $0.251-0.5$ & 2 & 9 (12.9) & $9.23 \pm 1.08$ & $1.21 \pm 1.63$ & $10.76 \pm 1.33$ \\
\hline $0.51-1$ & 3 & $15(21.4)$ & $15.43 \pm 2.47$ & $20.98 \pm 4.84$ & $18.73 \pm 2.77$ \\
\hline $1.01-1.5$ & 4 & $9(12.9)$ & $17.54 \pm 0.87$ & $23.41 \pm 2.22$ & $21.43 \pm 2.5$ \\
\hline \multirow[t]{2}{*}{$1.51-1.9$} & 5 & $15(21.4)$ & $20.08 \pm 1.54$ & $25.83 \pm 2.36$ & $25.09 \pm 3.76$ \\
\hline & Total & 70 & $13 \pm 5.3$ & $17.3 \pm 7.19$ & $15.97 \pm 6.64$ \\
\hline
\end{tabular}

SD: Standard deviation, BSA: Body surface area, Asc Ao: Ascending aorta

Table 4. Indexed aortic measurements

\begin{tabular}{|c|c|c|c|c|c|}
\hline $\operatorname{BSA}\left(\mathrm{m}^{2}\right)$ & Group & n (\%) & $\begin{array}{l}\text { Aort annulus } / \mathrm{m}^{2} \\
\text { Mean } \pm \text { SD }\end{array}$ & $\begin{array}{l}\text { Sinus of valsalva/m² } \\
\text { Mean } \pm \text { SD }\end{array}$ & $\begin{array}{l}\text { Asc Ao/m² } \\
\text { Mean } \pm \text { SD }\end{array}$ \\
\hline $0-0.25$ & 1 & $22(31.46)$ & $33.06 \pm 4.7$ & $44.61 \pm 7.14$ & $42.37 \pm 7.81$ \\
\hline $0.251-0.5$ & 2 & $9(12.9)$ & $26.97 \pm 2.88$ & $35.3 \pm 3.89$ & $31.44 \pm 3.66$ \\
\hline $0.51-1$ & 3 & $15(21.4)$ & $19.59 \pm 2.94$ & $26.79 \pm 6.59$ & $24.12 \pm 5.56$ \\
\hline $1.51-1.9$ & Total & 70 & $22.77 \pm 8.8$ & $30.51 \pm 12.33$ & $28.3 \pm 11.92$ \\
\hline
\end{tabular}


Table 5. Patients with Z-score $>2$

\begin{tabular}{|l|l|l|l|l|l|}
\hline Patients & $\begin{array}{l}\text { Age } \\
\text { (year) }\end{array}$ & Sex & $\begin{array}{l}\text { Z-score } \\
\text { Ao ann }\end{array}$ & $\begin{array}{l}\text { Z-score } \\
\text { SoV }\end{array}$ & $\begin{array}{l}\text { Z-score } \\
\text { Asc ao }\end{array}$ \\
\hline EC & 6.00 & Female & 2.80 & - & - \\
\hline BYK & 6.50 & Male & 4.19 & 6.37 & 2.67 \\
\hline YAD & 6.00 & Male & - & 3.17 & 4.53 \\
\hline CPB & 7.75 & Male & - & 2.09 & - \\
\hline NU & 18.00 & Female & - & - & 2.18 \\
\hline YA & 17.50 & Male & - & - & 2.73 \\
\hline FI & 0.04 & Female & 2.98 & - & - \\
\hline PA & 0.01 & Female & - & - & 2.48 \\
\hline KIS & 0.02 & Male & - & - & 2.30 \\
\hline Ao & & & & & \\
\hline
\end{tabular}

Ao ann: Aortic annulus, SoV: Sinus of valsalva, Asc ao: Ascending aorta

\section{Discussion}

As aort pathologies can be seen with isolated or complicated heart diseases in children, they can also accompany vasculitis (like Takayashu arteritis, Kawasaki disease, Behçet's disease) and genetic connective tissue disorders (Vascular form of Ehlers-Danlos, Marfan sydrome and Tuberous sclerosis). It has made us review the importance of the situation due to the significant mortality and morbidity seen in the influenced individuals. It is estimated that the frequency of aneurysm is 5.9/100000 patients per year in adults. Although it is estimated that the frequency is lower in children, there are no data for that ${ }^{(7,8)}$.

Cantinotti et al. ${ }^{(3)}$ have performed 2D echocardiography and annulus and vascular diameter measurements in their study conducted on 1151 healthy Caucasian Italian children with the mean age of 23 months and with BSA $0.54 \mathrm{~m}^{2}$. In our study, the mean age was 5.68 years and BSA was $0.75 \mathrm{~m}^{2(3)}$.

In Cantinotti et al. ${ }^{(3)}$ 's nomograms, the mean Ao ann was $13.48 \mathrm{~mm}$, SoV was $18.4 \mathrm{~mm}$ and Asc ao was 16.6 $\mathrm{mm}$ according to the BSA of $0.75 \mathrm{~m}^{2}$. These values were measured as $13 \mathrm{~mm}, 17.3 \mathrm{~mm}$ and $15.97 \mathrm{~mm}$, respectively, in our study. These were similar to the values that Cantinotti et al. ${ }^{(3)}$ reported.
With an average Ao ann Z-score value of 0.11 for the mean BSA value of $0.75 \mathrm{~m}^{2}$, nomogram Ao ann value determined by Cantinotti et al. ${ }^{(3)}$ was $13.5 \mathrm{~mm}$ while it was $13 \mathrm{~mm}$ in our study. Similarly, with an average SoV Z-score value of 0.28 for the mean BSA value of $0.75 \mathrm{~m}^{2}$, nomogram SoV value determined by Cantinotti et al. ${ }^{(3)}$ was $16.66 \mathrm{~mm}$ while it was $17.3 \mathrm{~mm}$ in our study.

As the findings above, with an average Asc Ao Z-score value of 0.10 for the mean BSA value of $0.75 \mathrm{~m}^{2}$, nomogram Asc Ao Z-score value reported by Cantinotti et al. ${ }^{(3)}$ was $17 \mathrm{~mm}$ while it was $15.97 \mathrm{~mm}$ in our study.

The mean gestational age was 29.8 weeks, weight was $1479 \mathrm{~g}$, BSA was $0.13 \mathrm{~m}^{2}$ in the study of Abushaban et al. ${ }^{(9)}$, in which they reported reference intervals for aortic diameter in preterm infants ${ }^{(9)}$. Asc Ao diameter for the mean BSA value was $7.5 \mathrm{~mm}$. This value is also in accordance with the mean aortic diameter value of 8.78 $\mathrm{mm}$ in patients in group 1. There was a 34-35-week-old patient with intrauterin growth retardation in our study. While Z-score Asc Ao value given for the mean BSA value of $0.13 \mathrm{~m}^{2}$ was between- 1.79 and +1.37 , the mean Asc Ao Z-score value for group 1 was -0.40 in our study.

Kervancioglu et al. ${ }^{(10)}$ assessed aortic root diameter in 229 healthy children through M-mode echocardiography. They reported that the mean aortic root diameter was 14.8 $\mathrm{mm}$ and the mean indexed aortic root diameter was 19.9 $\mathrm{mm} / \mathrm{m}^{2}$.

It is known that indexed diameter is (diameter/BSA) also important just like the diameter of aortic segment in normal children and adults. The maximum level of the indexed aortic root is $>21 \mathrm{~mm} / \mathrm{m}^{2}$; significant dilatation $>25 \mathrm{~mm} / \mathrm{m}^{2}$; meaningful risk for rupture $>27.5 \mathrm{~mm} /$ $\mathrm{m}^{2(11,12)}$. Z-score value defined for mild aortic dilatation is 1.9-3 and 3-4 for moderate dilatation ${ }^{(13)}$.

Chong et al. ${ }^{(14)}$ studied on 67 children with operated Tetralogy of Fallot whose mean age was $8.3 \pm 5.6$ years. They reported the prevalence of aortic dilatation (Z-score $>2$ ) as $88 \%$ for aortic annulus, $87 \%$ for sinus of valsalva, $61 \%$ for sinotubular junction, and $63 \%$ for ascending aorta. 
In our study, the mean indexed ao annulus value was $22.77 \mathrm{~mm} / \mathrm{m}^{2}$, sinus of valsalva value was $30.51 \mathrm{~mm} / \mathrm{m}^{2}$, and ascending aortic value was $28.30 \mathrm{~mm} / \mathrm{m}^{2}$.

Three of nine patients with Z-score values of $>2$ were the patients who were diagnosed, followed-up, and treated previously. Six of them were the patients who were assessed echocardiographically for the first time. The mean aortic annulus value for nine patients whose Z-scores were $>2$ was $26.5 \mathrm{~mm} / \mathrm{m}^{2}$, sinus of valsalva value was $38.24 \mathrm{~mm} / \mathrm{m}^{2}$, and ascending aortic value was $34.94 \mathrm{~mm} / \mathrm{m}^{2}$. Two of the patients with Z-scores $>3$ were diagnosed previously. Z-score aortic annulus and sinus of valsalva values for a 6.5 -year-old boy patient were 4.19 and 6.37, respectively. Z-score SoV and ascending aortic values for a 6-year- old boy patient were 3.17 and 4.53, respectively. Seven patients with Z-scores between 2 and 3 were first time visiting patients.

\section{Conclusion}

In the present study, children aged between 0 and 18 years, the mean aortic annulus, sinus of valsalva and ascending aorta, Z-scores and indexed values (aortic annulus $/ \mathrm{mm}^{2}$, sinus of valsalva $/ \mathrm{m}^{2}$, ascending aorta $/ \mathrm{m}^{2}$ ) were determined.

In this cross-sectional study including a few patients, aortic dilatations were observed in the measurements of six of 67 patients $(8.9 \%)$ who visited for the first time and in nine of 70 patients in total (12\%).

It is remarkable that non-syndromic newborn and infant patients who are thought to be healthy have also various levels of aortic dilatations even before they are diagnosed with aortopathy. We believe that the findings and results obtained in this cross-sectional study will lead to larger studies with patients with/without congenital heart diseases and syndromic/non-syndromic patients.

\section{Ethics}

Ethics Committee Approval: The center where I worked was changed (Merkezefendi State Hospital to Manisa City Hospital) one week after the end of the study.
Ethics committee approval could not be obtained because these institutions were not educational and research hospitals.

Informed Consent: Informed consent was taken from the patients.

Peer-review: Externally and internally peer-reviewed.

Financial Disclosure: The author declared that this study received no financial support.

\section{References}

1. Francois K. Aortopathy associated with congenital heart disease: A current literature review. Ann Pediatr Cardiol 2015;8:25-36.

2. Zanjani KS, Niwa K. Aortic dilatation and aortopathy in congenital heart disease. J Cardiol 2013;61:16-21.

3. Cantinotti M, Giordano R, Scalese M, et al. Nomograms for twodimensional echocardiography derived valvular and arterial dimensions in Caucasian children. J Cardiol 2017;69:208-15.

4. Lai WW, Gewa T, Shirali GS, et al. Guidelines and standards for performance of a pediatric echocardiogram: A report from the Task Force of the Pediatric Council of the American Society of Echocardiography. J Am Soc Echocardiogr 2006;19:1413-30.

5. Lopez L, Colan SD, Frommelt PC, et al. Guidelines and Standards Recommendation for Quantification Methods During the Performance of a Pediatric Echocardiogram: A Report From the Pediatric Measurements Writing Group of the American Society of Echocardiography Pediatric and Congenital Heart Disease Council. J Am Soc Echocardiogr 2010;23:46595.

6. Colan SD. The way and how of Z scores. J Am Soc Echocardiogr 2013;26:38-40.

7. Bickerstaff LK, Pairolero PC, Hollier LH, et al. Thoracic aortic aneurysms: a population-based study. Surgery 1982;92:1103-8.

8. Puranik R, Chow CK, Duflou JA, et al. Sudden death in the young. Heart Rhythm 2005;2:1277-82.

9. Abushaban L, Mariappa TV, Rathinasamy J, et al. Normal reference for aortic diameters in preterm infants. J Saudi Heart Assoc 2018;30:86-94.

10. Kervancioglu P, Kervancioglu M, Tuncer CM. Echocardiographic study of aortic root diameter in healthy children. Saudi Med J 2006;27:27-30.

11. Roman MJ, Devereux RB, Kramer-Fox R, et al. Two-dimensional echocardiographic aortic root dimensions in normal children and adults. Am J Cardiol 1989;64:507-12.

12. Davies RR, Gallo A, Coady MA, et al. Novel measurement of relative aortic size predicts rupture of thoracic aortic aneurysms. Ann Thorac Surg 2006;81:169-77

13. Lang RM, Bierig M, Devereux RB, et al. Chamber Quantification Writing Group; American Society of Echocardiography's Guidelines and Standards Committe; European Association of Echocardiography. Recommendations for chamber quantification: A report from the American Society of Echocardiography's Guidelines and Standards Committee and the Chamber 
Quantification Writing Group, developed in conjunction with the European Association of Echocardiography, a branch of the European Society of Cardiology. J Am Soc Echocardiogr 2005;18:1440-63.
14. Chong Wy, Wong WHS, Chiu CSW, Cheung YF. Aortic Root Dilation and Aortic Elastic Properties in Children After Repair of Tetralogy of Fallot. Am J Cardiol 2006; 97:905-9. 